
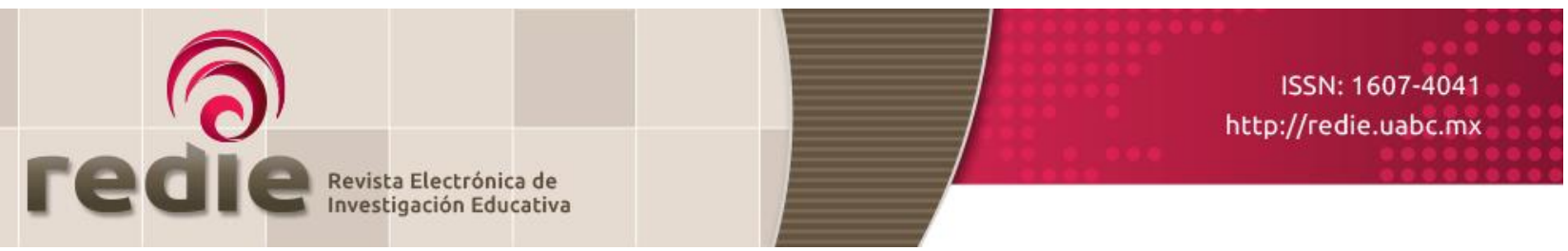

Vol. 21, 2019/e24

\title{
Asistencia y deserción escolar de la juventud indígena en Secundaria
}

\section{Attendance and Dropout among Indigenous Junior High Students}

Ivania de la Cruz Orozco (*) ivania.delacruz@cide.edu

Blanca Heredia Rubio (*) heredia.blanca@gmail.com

(*) Centro de Investigación y Docencia Económicas

(Recibido: 11 de mayo de 2017; Aceptado para su publicación: 25 de septiembre de 2017)

Cómo citar: De la Cruz, I. y Heredia, B. (2019). Asistencia y deserción escolar de la juventud indígena en Secundaria. Revista Electrónica de Investigación Educativa, 21, e24, 1-11. doi:10.24320/redie.2019.21.e24.1973

\section{Resumen}

El artículo presenta evidencia sobre algunos factores que inciden en la deserción y asistencia escolar de estudiantes indígenas de secundaria. Primero, se diseñó un modelo para estimar los factores que inciden en la brecha de deserción usando la Encuesta Nacional de Ingresos y Gastos de Hogares. Se encontró que uno de los elementos que más incide en la deserción escolar de estudiantes indígenas es la lengua. Posteriormente, se realizó investigación cualitativa en secundarias de Yucatán y Chiapas. Los hallazgos apuntan a que los estudiantes indígenas que ingresan a la secundaria transitan a un entorno donde el uso de su lengua se reduce o desaparece y enfrentan dificultades para adaptarse, estas dificultades afectan su desempeño y desembocan en abandono escolar.

Palabras clave: Deserción escolar, asistencia escolar, poblaciones indígenas, minoría lingüística, enseñanza secundaria.

\section{Abstract}

This article presents evidence of some factors affecting school dropout and attendance in indigenous students in junior high schools. First, a model was designed to estimate factors that affect the dropout gap using the National Household Income and Expenditure Survey. It was found that language is one of the main aspects affecting school dropout among indigenous students. A qualitative study was then conducted in junior high schools in Yucatán and Chiapas. The findings suggest that indigenous students who enter junior high school transition into an environment where the use of their language is reduced or non-existent, and they struggle to adapt. These difficulties affect their performance and ultimately lead them to drop out of school. 


\section{Introducción}

El sistema educativo mexicano opera en entornos socioeconómicos y culturales muy diversos. A pesar de contar con importantes avances en materia de cobertura, la autoridad educativa en México no ha logrado adecuar contenidos, materiales, equipo e infraestructura para atender de manera pertinente a una población social, étnica y lingüísticamente diversa. Esto se ha traducido en fuertes desigualdades educativas para los sectores más desfavorecidos, siendo las poblaciones indígenas y rurales las que, consistentemente, han padecido mayor rezago en cuanto a la calidad de la educación que reciben y de los resultados que obtienen (INEE y UNICEF, 2016). En México hay alrededor de 4 millones de niños y adolescentes indígenas en edad escolar, y se calcula que 1.8 millones habla alguna lengua indígena, aunque no hay datos precisos sobre su nivel de competencia en dicha lengua. Independientemente de ello, esta población tiene el derecho a recibir educación en su idioma (INEE y UNICEF, 2016).

La experiencia y logro escolar de la juventud indígena en México están en desventaja frente a las de la población no indígena. La evidencia disponible indica que existe una brecha educativa del 34\% entre la población indígena y no indígena en México (Chávez-Herrería, Abreu y Charles, 2013). Lo anterior significa que el logro educativo -compuesto por indicadores de asistencia, escolaridad, alfabetismo y desempeñode la población indígena en el país es equivalente al $76 \%$ del logro educativo de la población no indígena. Si bien estos datos son reveladores, la evidencia disponible no alcanza a señalar cuáles son los factores que inciden en la brecha total ni en los elementos que la componen.

Esta laguna en los estudios académicos puede deberse a la falta de indicadores demográficos y escolares para las distintas comunidades indígenas del país. Lo anterior constituye un obstáculo para el mejor entendimiento de las desventajas y fortalezas escolares de la niñez y juventud indígenas. Asimismo, dificulta el diseño e implementación de intervenciones efectivas para disminuir la brecha educativa entre las poblaciones indígena y no indígena.

Esta investigación busca contribuir a la generación de evidencia sobre los factores que inciden en dos indicadores del logro educativo: deserción y asistencia escolar, particularmente para el nivel secundaria. El estudio se acotó de esta forma por tres razones:

Primero, se decidió enfocar el análisis en la asistencia escolar debido a que este indicador es notablemente inferior para la población indígena a partir de la transición de primaria a secundaria. Así, la asistencia a la escuela de la población no indígena en edad de cursar secundaria decrece casi 5 puntos respecto a la tasa de asistencia que tiene la población no indígena en edad de cursar primaria. En contraste, dicha diferencia en asistencia escolar es de 10 puntos para la población indígena y de 13 puntos para la población hablante de lengua indígena (Chávez-Herrería et al. 2013; INEE y UNICEF, 2016). Además, en 2014 el 94.3\% de la población de entre 12 y 14 años asistía a la escuela, pero para adolescentes hablantes de español y lengua indígena esta cifra fue de $87.4 \%$, mientras que para los monolingües en lengua indígena la cifra fue de 83.1\% (INEE y UNICEF, 2016). Si bien hay estudios que exploran los factores familiares, económicos y sociales asociados con la experiencia y trayectoria escolar de niños y jóvenes indígenas (UNICEF, 2016), dichos estudios no permiten un análisis de toda la población indígena en edad escolar frente a la no indígena.

Segundo, la mayoría de los estudios sobre educación a pueblos originarios se concentra en el nivel primaria. Lo anterior puede deberse a que para dicho nivel se tienen programas federales diseñados para comunidades indígenas, pero para el nivel secundaria no existe un programa equivalente para que atienda a la población monolingüe en o hablante de lengua indígena: se asume que todos los egresados de primaria podrán tomar clases en español. Así, para el nivel secundaria el currículo está en español y los docentes suelen ser de comunidades distintas a donde se ubican las escuelas, no han sido formados como maestros de educación indígena y no hablan la lengua de la comunidad (Fernández, Torres y García, 2016). Se estima que en 2015 había 5,000 estudiantes monolingües en lengua indígena que asistían a los distintos tipos de servicio en secundaria. De los estudiantes que hablan tanto lengua indígena como español en edad de cursar secundaria, se sabe que $52.4 \%$ asistía a telesecundarias, $23.3 \%$ a secundarias técnicas y $21.4 \%$ a secundarias generales (INEE y UNICEF, 2016, p. 10). Además, el $77.6 \%$ de las secundarias con al menos un estudiante que habla lengua indígena sólo cuenta con maestros que hablan español. En 
escuelas donde los maestros hablan una lengua indígena, sólo en el 38.5\% de ellas los docentes hablan la misma lengua que hablan todos los estudiantes (INEE y UNICEF, 2016).

Tercero, el enfoque de esta investigación en la educación secundaria se debe a que se trata de un momento decisivo para los estudiantes, quienes viven una doble transición. Por un lado, acceden a un nivel educativo con mayor grado de dificultad y con mayor diversidad de contenidos. Por otro lado, en este nivel los estudiantes pasan de la niñez a la adolescencia, etapa caracterizada por múltiples cambios cognitivos, emocionales, sociales y sexuales. Es en estos años cuando los jóvenes enfrentan factores de riesgo como la drogadicción y el embarazo adolescente. Como afirman Barrón y Ducoing (2017), al considerarse parte de la educación básica junto con la educación primaria, la secundaria está contextualizada y orientada a una cultura escolar que no se enfoca en la etapa que viven sus estudiantes: la transición a la vida adulta. Así, al asociar a la secundaria con la primaria se minimizan los procesos de definición de identidad y procesos emocionales que emergen durante la adolescencia (Barrón y Ducoing, 2017).

Por las razones mencionadas, esta investigación tiene por objetivo establecer los factores que inciden en la brecha de deserción en secundaria que se da entre la población indígena y no indígena en México. Para medir el efecto de dichos factores, la investigación contó con una sección cuantitativa; y para entender las características y formas de dichos efectos se realizó investigación cualitativa en Chiapas y Yucatán.

\subsection{Antecedentes sobre asistencia y deserción escolar de la juventud indígena en secundaria}

México, y la mayoría de países de América Latina, tienen una tasa de transición de primaria a secundaria por arriba del 90\%. Sin embargo, casi una tercera parte de la población en edad de cursar educación secundaria está fuera de la escuela (Barrón y Ducoing, 2017). Esto pudiera deberse al fenómeno del abandono escolar en el nivel secundaria. En el caso de México, se sabe que el nivel de matriculación en secundaria para el año 2014-2015 en secundaria fue de 96\%, pero hubo 272,372 estudiantes que no concluyeron sus estudios, resultando en una tasa de deserción de 4\% (INEE, 2016).

Respecto a los factores asociados al abandono escolar, resaltan los factores económicos y culturales (UNICEF, 2016), aunque también se debe considerar el efecto de la estructura y orientación de las instituciones encargadas de brindar el servicio educativo (Barrón y Ducoing, 2017; Tapia, Pantoja y Fierro, 2010). Para la década de los noventa, Arenas, Rubalcava y Teruel (2016) encontraron que la terminación del nivel secundaria y la transición a educación media superior estaba asociada con los años de escolaridad de los padres, con la no ausencia de madre y padre, y con un número menor de hermanos, favoreciendo más a la escolaridad de mujeres que de varones (Arenas et al., 2016). Para el período entre 2002 y 2006, Gibbs e Heaton (2014) analizaron la deserción entre primaria y secundaria en México y encontraron que la población que habita zonas rurales y que habla una lengua indígena presenta mayor riesgo de deserción. Asimismo, los autores reportan mayor riesgo de deserción para familias de padres ausentes, padres que se dedican al trabajo agrícola, padres desempleados y cuando los estudiantes deben trabajar. Gibbs e Heaton (2014) encontraron que dos variables tienen un peso menor en el riesgo de deserción en secundaria frente al peso que tienen en la probabilidad de desertar en primaria: años de escolaridad de los padres y reprobación (Gibbs e Heaton, 2014). En un estudio más reciente, Vargas y Valadez (2016), dan información sobre la brecha en la probabilidad de deserción por grupo de edad, nivel socioeconómico y calidad percibida de la última escuela (Vargas y Valadez, 2016). Sin embargo, no se explora la brecha en deserción entre estudiantes indígenas y no indígenas.

\section{Método}

Respecto a la deserción escolar, se busca precisar la magnitud del efecto de ciertos factores durante secundaria a partir de la Encuesta Nacional de Ingresos y Gastos de los Hogares 2013 (ENIGH) (INEGI,

\footnotetext{
${ }^{1}$ Cabe señalar que estos datos provienen del CEMABE (Centro de Escuelas, Maestros y Alumnos de Educación Básica y Especial), el cual no pudo levantar datos de todas las escuelas en Chiapas, Oaxaca y Michoacán (INEGI, 2013). Así, las cifras presentadas no reflejan la situación de estos estados con alta concentración de población indígena.
} 
2013b), la cual cuenta con representatividad a nivel nacional y municipal, y para población indígena. Si bien la ENIGH se aplicó en todas las entidades de la república, en varias de ellas la información sobre el estatus lingüístico es incompleta, esto es, no se indica si los hablantes de lengua indígena son monolingües o no. Dado que dicha variable es de enorme importancia en este análisis, se decidió reducir la muestra a los estados con datos completos: Campeche, Chiapas, Chihuahua, Hidalgo, Oaxaca, Puebla, Quintana Roo, San Luis Potosí, Tlaxcala y Yucatán. Así, los resultados son representativos de estas entidades.

Se incluyeron variables que tienen que ver con el estatus lingüístico de las personas: i) si ellas y sus padres hablan lengua indígena o no; ii) si los padres son monolingües en esta lengua, y iii) si se autoadscriben como indígenas. Se controla por posibles problemas de endogeneidad con el método de variables instrumentales. Siguiendo el planteamiento de Parker, Rubalcava y Teruel (2005), en este estudio, el instrumento que se utiliza es el estatus lingüístico de la madre, cuya relevancia está sustentada en la literatura (Arenas et al., 2016). Aunque los estudiantes sean bilingües y el español sea la lengua de instrucción en la escuela, el que la madre hable una lengua indígena significa que el estudiante emplea la lengua indígena en importantes procesos de socialización en los entornos familiar y comunitario (Mier y Terán y Rabell, 2013).

En cuanto a la variable dependiente, el indicador de permanencia que se utiliza está definido de manera que $1=$ el estudiante desertó durante la educación secundaria y $0=$ no desertó. En resumen, los modelos estiman el efecto de ser un estudiante inicialmente monolingüe hablante de una lengua indígena en la probabilidad de desertar de la escuela durante la secundaria.

En los modelos estadísticos se utilizan como variables de control aquellos factores que, según la literatura consultada para esta investigación, inciden en la deserción: escolaridad de los padres, indicadores socioeconómicos, sexo y edad. Respecto de la medida del nivel socioeconómico, se eligió construir y utilizar el Estatus Económico, Social y Cultural (ESCS, por sus siglas en inglés) definido por la oCDE para analizar los resultados de la prueba PISA 2012 -medidor validado internacionalmente que permite controlar por diversos factores eficientemente. El índice incorpora valoraciones sociales de la ocupación del padre y de la madre, así como su escolaridad y posesiones del hogar que se relacionan con el estatus cultural y económico, combinándolos en una sola variable mediante el análisis de componentes principales. Además, en todos los modelos se controló por el sexo y la edad de los estudiantes y por el nivel de marginación de la comunidad.

En un primer momento se usaron modelos de probabilidad lineal para probar hipótesis sobre los efectos de ser indígena de acuerdo con diferentes definiciones de "indígena". Asimismo, estos modelos sirven para validar, de cierta forma, la relevancia del instrumento. Posteriormente, se utilizaron modelos de efectos fijos con los errores agrupados a diferentes niveles geográficos (localidad, municipio y estado) en un modelo de máxima verosimilitud (logit). Luego, se repitió el procedimiento para obtener resultados de los estados para los que está disponible la información. Por último, se desagregó la variable dependiente en los tres grados de secundaria y se verificaron los efectos de las variables de interés con un modelo econométrico igual a los anteriores. Se añadió también una breve prueba de los efectos de las escuelas de atención indígena al final del ejercicio.

La sección cualitativa de esta investigación tuvo por objetivo profundizar en los factores que afectan la asistencia escolar de la población indígena. Como se mencionó en párrafos anteriores, el análisis cuantitativo con datos de la ENIGH se limitó a aquellas entidades para las cuales había información completa para construir las variables relevantes del modelo. El apartado cualitativo del estudio se centró en dos de estas entidades: Yucatán y Chiapas. Se eligieron dichas entidades porque en ellas tiene presencia la agencia que financió la investigación (Fundación W. K. Kellogg). Posteriormente, se identificaron municipios donde hubiera una brecha en asistencia a secundaria de al menos $35 \%$ entre población indígena y no indígena. La selección de escuelas se basó en el criterio de accesibilidad: se hizo trabajo de campo en secundarias que dieron autorización para realizar entrevistas y aplicar cuestionarios a estudiantes, padres de familia y maestros. En su fase cualitativa el estudio contó con la participación de 134 informantes. 
Como se verá más adelante, los hallazgos de la sección cuantitativa brindan un primer diagnóstico sobre la deserción entre estudiantes indígenas en México. Sin embargo, los datos disponibles para el estudio cuantitativo poco nos dicen de las prácticas docentes, de la convivencia entre integrantes de la comunidad escolar, de las valoraciones de desempeño estudiantil por parte de los maestros, de las formas de involucramiento familiar en la escuela, de la lengua o lenguas usadas por maestros y estudiantes en el recinto escolar, entre otros. El objetivo del estudio cualitativo realizado fue profundizar justamente en dichos factores, los cuales afectan la asistencia a secundaria de la población indígena.

La recolección de información se realizó con investigadores de apoyo, quienes tenían experiencia trabajando en las comunidades incluidas en el estudio. Se aplicó un cuestionario a toda la comunidad escolar y entrevistas semi-estructuradas. Siempre que fue posible se recogieron las percepciones de directivos, docentes, estudiantes y padres de familia. La tabla I presenta fechas del levantamiento en campo y la distribución de entrevistas por entidad y tipo de actor.

Tabla I. Trabajo de campo

\begin{tabular}{l|l|c|c|c|c|c}
\hline \multicolumn{1}{c|}{ Municipio $^{2}$} & \multicolumn{1}{|c|}{$\begin{array}{c}\text { Levantamiento } \\
\text { en campo }\end{array}$} & Directores & Maestros & Estudiantes & $\begin{array}{c}\text { Padres } \\
\text { de familia }\end{array}$ & Total \\
\hline Yucatán & enero a junio 2015 & 1 & 5 & 34 & 12 & 52 \\
Yucatán & enero a junio 2015 & - & 4 & 26 & 7 & 37 \\
Chiapas & diciembre 2015-enero 2016 & - & $8^{3}$ & 30 & 7 & 45 \\
Total & & 1 & 17 & 90 & 26 & 134 \\
\hline
\end{tabular}

Todas las entrevistas fueron audiograbadas para garantizar el anonimato de los participantes. La mayoría de las entrevistas realizadas a estudiantes y padres de familia en Chiapas se hicieron en tseltal y posteriormente fueron traducidas al español.

El análisis de información se organizó de acuerdo con cuatro grandes categorías de factores. Los factores individuales se refieren a las propiedades personales que distinguen a los estudiantes al momento de estudiar educación secundaria, como edad, género, grupo étnico, opinión que tienen sobre sí mismos y sobre su adaptación a ese nivel educativo, su situación laboral, sus aspiraciones escolares y elementos que caracterizaron su experiencia escolar previa. Los factores del entorno familiar hacen referencia a características del hogar y la familia de los estudiantes; entre ellos se encuentran el nivel socioeconómico del hogar, el nivel educativo de los padres, la disponibilidad de materiales escolares en el hogar y las aspiraciones escolares que los padres tienen de sus hijos. La información de esta sección fue recabada a través de entrevistas con padres y madres de familia. Los factores escolares reflejan características de las secundarias a las que acuden los estudiantes. Así, en esta categoría se describen aspectos como infraestructura, equipamiento y materiales escolares, modalidad educativa y localización de las escuelas, características de la relación alumno-maestro, particularidades del ambiente escolar y las condiciones de la labor docente. Finalmente, los factores de la comunidad incluyen propiedades de los lugares donde se ubican los centros escolares, como la disponibilidad de servicios básicos, las actividades económicas que se realizan y los factores vinculados a la discriminación estructural que enfrentan las localidades con población indígena, las cuales perpetúan su condición de exclusión económica y social. Esta categorización se basó en literatura que reconoce la multiplicidad de factores que inciden en la experiencia escolar, más allá de las características del estudiante (Tapia, Pantoja y Fierro, 2010).

\section{Resultados}

\subsection{Deserción}

Se utilizaron los datos de la ENIGH, correspondientes a seis entidades federativas, para determinar si la probabilidad de que un joven estudiante abandone la escuela secundaria era más alta si se trataba de un

\footnotetext{
${ }^{2}$ Se omitieron los nombres de los municipios para garantizar la confidencialidad de los participantes.

${ }^{3}$ Se entrevistó a 3 maestros de la secundaria en donde se hizo trabajo de campo y a 5 maestros de otros niveles educativos en la región de los Altos de Chiapas.
} 
hablante de lengua indígena. Son cinco los hallazgos principales: 1) se encontró que la brecha relativa a deserción es más alta en Puebla, seguida por Chiapas, Tlaxcala y Yucatán. Sólo en Hidalgo y Oaxaca es menos probable que un joven abandone la secundaria si es indígena; 2) se observó que los factores que más inciden en la deserción son las características de los municipios y no las de los estados. Una hipótesis es que están ligadas a los sistemas políticos locales y a la política educativa municipal; 3) la mayoría de las deserciones ocurre en el segundo grado de secundaria. Es en este grado donde la diferenciación en deserción de los indígenas crece más con respecto a la de los no indígenas (se abre la brecha); 4) se encontró que los individuos que se identifican como indígenas son $2 \%$ más propensos a abandonar la escuela que los que no se identifican como indígenas, independientemente de su condición socioeconómica o la de su comunidad. Además, si un individuo habla lengua indígena, es $4 \%$ más probable que abandone la secundaria, independientemente de su condición socioeconómica o la de su comunidad. Por último, se encontró que si la madre de un estudiante habla sólo la lengua indígena, el estudiante es $10 \%$ más propenso a abandonar la secundaria, independientemente de su condición socioeconómica o la de su comunidad.

Los hallazgos del análisis cuantitativo apuntan a que el elemento que más incide en el desempeño y la escolaridad de los estudiantes indígenas es la lengua.

\subsection{Asistencia a la escuela (estudiantes de Yucatán y Chiapas)}

A continuación se presentan los resultados enmarcados en las categorías de análisis antes mencionadas.

Factores individuales: El conjunto de estudiantes entrevistados consideraba que su experiencia educativa en primaria había sido positiva, que su desempeño escolar era bueno y que les gustaba asistir a la primaria. Para muchos estudiantes, la transición de la primaria a la secundaria no resultó un cambio sencillo debido a las distintas dinámicas que, en su opinión, implicaba asistir a otro nivel educativo: aumento en el número de maestros, de materias y de carga de trabajo. Aunque los estudiantes entrevistados refirieron haberse adaptado y sentirse a gusto en la secundaria, en las escuelas de ambos estados muchos jóvenes reportaron dificultades con materias como Matemáticas, Español e Inglés.

Estas dificultades se agudizaban aún más cuando se trataba de estudiantes con poco dominio del español, quienes al ingresar a secundaria enfrentaban serias limitaciones para comunicarse con los maestros y otros compañeros, para entender y participar en las clases y para realizar sus tareas. De hecho, hubo estudiantes que externaron sentir vergüenza de hablar y participar en clase, y que habían sido objeto de burla por parte de sus compañeros. Además, varios estudiantes provenían de hogares monolingües en lengua indígena, lo cual reducía su exposición al español e influía en su desempeño escolar. Lo anterior fue particularmente relevante para el caso de la secundaria en Chiapas. Independientemente de las dificultades asociadas al uso del español como única lengua de instrucción, se encontró que la falta de aspiraciones escolares no es un factor de riesgo, ya que casi todos los entrevistados en Chiapas y Yucatán deseaban continuar con sus estudios y conseguir una carrera técnica o licenciatura; la siguiente cita ejemplifica esta situación:

Mi dificultad en la secundaria es que como no hablante de la lengua castellano se me hace difícil expresarme y participar dentro de mi salón, también no sé escribir muy bien. (Estudiante de Chiapas)

Factores familiares: Los padres de familia en Chiapas y Yucatán tienen una alta valoración por la educación como una oportunidad para que sus hijos mejoren las condiciones de vida y aseguren mejores ingresos. Por ello, se dijeron dispuestos a apoyarlos para continuar sus estudios. A pesar de que consideraron que las becas y programas de asistencia facilitaban seguir estudiando y que habían aligerado la carga económica que conlleva tener hijos en la escuela, los padres afirmaron que la economía familiar precaria era el principal obstáculo para que los jóvenes continuaran con sus estudios, pues los costos asociados con la educación son altos - por ejemplo, compra de materiales, transportación, dinero рага alimentos y cooperaciones. Además, los gastos se incrementan conforme aumenta el nivel educativo, el número de hijos y cuando no hay oferta educativa dentro de la comunidad. Por lo anterior, los padres se ven en la necesidad de pedir a sus hijos que trabajen para contribuir al ingreso familiar. La 
siguiente cita permite entender mejor los gastos privados de la educación:

Pues ahorita lo económico, porque ahorita les marcan mucha tarea, que para copias, que para el ciber. Si está media hora, una hora y sus copias, a veces es más de 20 pesos. En mi caso, uno lo busca y hay, pero hay donde de plano estudian dos o tres, necesitan 100 pesos diario, a veces tienen dos en la secundaria y uno en bachiller, es mucho. (Madre de familia, Yucatán)

Otro hallazgo relevante respecto a los factores familiares que afectan asistencia y desempeño escolar en secundaria es la dificultad que tienen los padres para ayudar a sus hijos con las tareas, dados los bajos niveles de escolaridad que tienen. En Yucatán la mayoría de padres entrevistados tiene estudios de secundaria con trayectorias interrumpidas, mientras que en Chiapas cuentan con primaria incompleta. Además, en Chiapas varios padres dijeron ser monolingües en lengua indígena y tener dificultad para establecer una comunicación efectiva con los maestros.

Factores escolares: En Chiapas, la insuficiente cobertura educativa implica altos costos en tiempo y en transporte para los estudiantes y sus familias. Algunos jóvenes debían caminar hasta hora y media de su casa a la escuela, lo cual hacía que su asistencia a clases fuera irregular y constituía un factor para cambiar de escuela o dejar de estudiar.

En Yucatán los participantes comentaron que la infraestructura y equipamiento de las escuelas y las aulas era insuficiente y se encontraba en mal estado. Por ejemplo, se carecía de espacios para la recreación o de ventilación adecuada en los salones y había mobiliario inservible. Se afirmó que, a pesar de contar con espacios como la biblioteca y computadoras con acceso a Internet, su uso no era frecuente ni los alumnos podían disponer de ellos para realizar sus tareas:

En mi salón los ladrillos ya se echaron a perder y las sillas de mis compañeros se les quita la paleta, se mueven. Hay una niña que de plano no sirve su silla, la paleta donde escribe nomás le haces así y se cae... también, en los baños no hay papel, falta un poco más de higiene en la escuela. (Estudiante, Yucatán)

Asimismo, se señalaron condiciones relacionadas con los maestros que influían en los procesos de enseñanza y de aprendizaje, tales como: ausentismo docente, pagos tardíos, contratos temporales, excesiva rotación, asignación a zonas alejadas, desconocimiento de la lengua de la comunidad e impartición de materias en las que no eran especialistas. Una maestra en Yucatán explica:

El año pasado yo empecé a trabajar hasta principios de octubre, de Matemáticas llegaron dos maestras, luego que siempre no, se fueron. Después otro, un mes, dos meses los alumnos sin maestro. Vino un maestro, luego que siempre no. A fin de cuentas creo que pasaron cuatro o cinco maestros en un curso escolar, ¿qué van a aprender los alumnos? (Maestra, Yucatán)

Se halló una estrecha relación entre, por un lado, las formas en que los maestros daban su clase y la cantidad de tareas que dejaban y, por otro lado, el gusto y facilidad que tenían los alumnos por ciertas materias. Esto es, los estudiantes reportaban más facilidad y gusto por esas materias si percibían que los maestros explicaban bien, ponían diversas actividades, hacían entretenida o divertida la clase, favorecían la participación de los alumnos y eran pacientes para explicar y resolver las dudas. Por el contrario, los maestros que, en opinión de los estudiantes, eran aburridos, que no explicaban bien los contenidos o que no eran pacientes para explicar, influían negativamente en la motivación y gusto de los estudiantes por las materias.

Las relaciones que se establecen en la escuela con los maestros y con los compañeros influyen fuertemente en el gusto por asistir a la secundaria. En general, los estudiantes de Chiapas y Yucatán tenían una opinión positiva de sus maestros al considerar que la mayoría los trataba por igual y con respeto. La buena convivencia con los compañeros se mencionó como la principal razón por la que los jóvenes asistían a la secundaria. Por el contrario, los conflictos con los compañeros (en una de las secundarias de Yucatán) y "recibir apodos" (en Chiapas) eran aspectos que disminuían el gusto por la escuela y constituían un factor a mejorar. 
Otro aspecto de suma relevancia es el dominio del español. La mayoría de los estudiantes y padres entrevistados hablaban lengua indígena y muchos maestros únicamente español, lo que influía en el desempeño de los estudiantes, que no siempre entendían ni se expresaban con confianza en esa lengua.

En general, los maestros de los dos estudios de caso consideraron que los planes y currículum nacionales no siempre se adaptaban a las condiciones locales, lo cual reducía su pertinencia.

Los factores escolares presentados en este apartado apuntan a la inexistencia de una condición básica para que los estudiantes puedan asistir a secundaria, experimentarla como una etapa formativa y agradable, y concluirla. Tapia, García y Fierro (2010) y Van Dijk (2012) señalan que no puede responsabilizarse de la deserción a los jóvenes; la escuela y el sistema educativo que la sostiene debe garantizar las condiciones que faciliten la permanencia de los estudiantes en la escuela.

Factores de la comunidad: Los maestros y padres participantes en los estudios de caso externaron que la presencia cada vez más frecuente de fenómenos como la drogadicción, la violencia, el embarazo adolescente y la migración nacional e internacional (sobre todo en Yucatán) constituían factores de riesgo para que los jóvenes abandonaran la escuela. Una maestra explica la situación en una de las escuelas visitadas en Yucatán:

Los papás están en Estados Unidos y lo que ellos quieren es terminar su secundaria e irse... recientemente, una muchacha se salió faltando dos meses en tercer grado, que su mamá la vino a sacar, que le compró su pasaje y todo para que se vaya. (Maestra, Yucatán)

Los maestros valoraron que las características de las comunidades rurales e indígenas (pequeñas, tranquilas, con respeto por la autoridad) favorecían que los jóvenes fueran más tranquilos que los de zonas urbanas y contribuían a su permanencia en la escuela.

A diferencia de otras épocas donde se apoyaba al hijo mayor o a los varones para proseguir con los estudios, en Chiapas y Yucatán se encontró que la condición de género de las mujeres ya no parecía ser una limitante para su educación, pues los padres entrevistados afirmaron que buscaban apoyar en los estudios tanto a sus hijas como a sus hijos. Si bien el hallazgo para Chiapas y Yucatán es alentador, es necesario realizar investigación a nivel comunidad (no escuela) para saber si se trata de un fenómeno generalizado o sólo corresponde a la visión de los padres entrevistados, ya que hay literatura que sostiene que la condición de género de las mujeres sigue incidiendo negativamente en sus trayectorias escolares (Mier y Terán y Rabell, 2013).

Los resultados del análisis cualitativo apuntan a que son múltiples los factores que inciden en la asistencia y desempeño escolar de la población indígena. Sin embargo, el uso del español como lengua única de instrucción y la precariedad de la economía familiar son dos de los aspectos que más influyen. Los hallazgos también sugieren la necesidad de políticas educativas diferenciadas que respondan a las necesidades y características de las comunidades. A manera de reseña, la tabla II presenta los hallazgos cuantitativos y cualitativos: 
Tabla II. Resumen de hallazgos

\begin{tabular}{|c|c|c|}
\hline Sección & Indicador & Hallazgo \\
\hline \multirow{5}{*}{ Cuantitativa } & \multirow{5}{*}{ Deserción } & $\begin{array}{l}\text { La brecha es mayor en Puebla, seguida por Chiapas, Tlaxcala y Yucatán. Sólo en } \\
\text { Hidalgo y Oaxaca es menos probable que un joven abandone la secundaria si es } \\
\text { indígena. }\end{array}$ \\
\hline & & $\begin{array}{l}\text { Los factores que más inciden en la deserción son las características de los municipios } \\
\text { y no las de los estados. } \\
\text { La mayoría de las deserciones ocurre en el } 20 \text {. grado de secundaria. En este grado la } \\
\text { diferenciación en deserción de los indígenas crece más con respecto a los no } \\
\text { indígenas (se abre la brecha). }\end{array}$ \\
\hline & & $\begin{array}{l}\text { Los individuos que se identifican como indígenas son } 2 \% \text { más propensos a } \\
\text { abandonar la escuela que los que no se identifican como indígenas. }\end{array}$ \\
\hline & & $\begin{array}{l}\text { Si un individuo habla lengua indígena es } 4 \% \text { más probable que abandone la } \\
\text { secundaria, independientemente de su condición socioeconómica o la de su } \\
\text { comunidad. }\end{array}$ \\
\hline & & $\begin{array}{l}\text { Si la madre de un estudiante habla sólo la lengua indígena el estudiantes es } 10 \% \text { más } \\
\text { propenso a abandonar la secundaria. }\end{array}$ \\
\hline \multirow{7}{*}{ Cualitativa } & Individual & $\begin{array}{l}\text { La transición a secundaria requiere de ajustes importantes por parte de los } \\
\text { estudiantes. }\end{array}$ \\
\hline & Familiar & $\begin{array}{l}\text { El entendimiento del español afecta la capacidad de los padres para involucrarse } \\
\text { tanto como quisieran en la educación de sus hijos. Asimismo, se ve afectada la } \\
\text { comunicación entre padres y docentes, quienes suelen ser monolingües en español. } \\
\text { El ingreso familiar es el factor que genera un mayor riesgo de deserción. Los gastos } \\
\text { de enviar un hijo a la secundaria son elevados, incluso recibiendo apoyo de } \\
\text { programas asistencialistas. }\end{array}$ \\
\hline & \multirow{3}{*}{ Escolar } & $\begin{array}{l}\text { Los jóvenes indígenas tienen que hacer frente a un sistema educativo que no se } \\
\text { ajusta a sus características: en este nivel escolar, el español se vuelve la lengua única } \\
\text { de instrucción. Los materiales, clases, tareas, participaciones y exámenes son en una } \\
\text { lengua que, si bien entienden, no siempre hablan fluidamente. }\end{array}$ \\
\hline & & $\begin{array}{l}\text { Los integrantes de la comunidad escolar refieren la necesidad de mejorar la } \\
\text { infraestructura y el equipamiento de las escuelas para optimizar el servicio educativo } \\
\text { a los estudiantes. }\end{array}$ \\
\hline & & $\begin{array}{l}\text { Las áreas de oportunidad respecto del cuerpo docente son: ausentismo, pagos } \\
\text { tardíos, contratos temporales, excesiva rotación, asignación a zonas alejadas, } \\
\text { desconocimiento de la lengua de la comunidad e impartición de materias en las que } \\
\text { no son especialistas. }\end{array}$ \\
\hline & \multirow{2}{*}{ Comunitario } & $\begin{array}{l}\text { La drogadicción, la violencia, el embarazo adolescente y la migración nacional e } \\
\text { internacional son factores que inciden en la asistencia escolar. }\end{array}$ \\
\hline & & $\begin{array}{l}\text { La falta de cobertura escolar resulta en gastos de transporte y alimentación que } \\
\text { afectan económicamente a las familias. }\end{array}$ \\
\hline
\end{tabular}

\section{Discusión y conclusiones}

La asistencia a la escuela secundaria pareciera ser un tema resuelto en México. Los altos niveles de transición de primaria a secundaria y la expansión de cobertura brindan, en general, un panorama alentador. Sin embargo, un acercamiento a la experiencia en secundaria de las juventudes indígenas revela la existencia de factores que dificultan la asistencia y abonan al riesgo de deserción escolar.

El relato por parte de los estudiantes mayas y tseltales sobre su experiencia en secundaria deja ver que a las dificultades asociadas a este nivel escolar se suma una complicación mayor: que el sistema educativo sigue sin considerar que hablan una lengua distinta al español. Así, los estudiantes expresan dificultad y vergüenza al hablar y participar en clase. Los docentes refieren la dificultad de comunicarse con sus estudiantes hablantes de lengua indígena y de entender en qué medida dichos estudiantes pueden seguir las lecciones; todo esto apoyándose en material que, los mismos docentes reconocen, no es pertinente para los contextos indígenas en secundaria. De igual forma, los padres de estudiantes indígenas refieren que el seguimiento al desempeño escolar de sus hijos se dificulta por no hablar la misma lengua que los docentes. Así, lo que se entiende como "éxito escolar" de los estudiantes indígenas implica su adaptación y asimilación. 
La evidencia que emerge del trabajo directo con integrantes de comunidades escolares es consistente con los hallazgos del modelo estadístico: en los estados incluidos en la muestra, la probabilidad de deserción en secundaria para estudiantes indígenas es mayor que para sus contrapartes no indígenas.

A pesar de las políticas educativas más recientes que buscan promover una educación intercultural y bilingüe, éstas se concentran más en educación primaria. Así, es evidente que existen estrategias diferenciadas para los estudiantes indígenas que en primaria acceden a escuelas donde habría materiales en su lengua y docentes bilingües, pero en secundaria se enfrentan con un sistema discriminatorio.

Los hallazgos de esta investigación hacen evidente una política educativa que promueve la discriminación institucional, observable en la presencia limitada, casi insignificante, de secundarias indígenas en lugares con alta concentración de población perteneciente a pueblos originarios. Transformar el sistema de educación secundaria para darle un enfoque bilingüe-bicultural no es tarea sencilla. Los encargados de política educativa tendrían que considerar proyectos de rescate de lenguas locales, diseño y uso de materiales en lenguas indígenas, capacitación y mejor ubicación de maestros, entre otros. Sin embargo, mantener una oferta educativa asimilacionista seguirá teniendo serias repercusiones en los indicadores educativos de la niñez y la juventud indígena. Como afirman Barrón y Ducoing (2017, p. 18), "la obligatoriedad del nivel secundaria requiere de propuestas curriculares que consideren la diversidad del estudiantado".

Los resultados obtenidos evidencian la necesidad de llevar a cabo más estudios al menos sobre tres temas: primero, se requiere investigación, de mayor profundidad y alcance, sobre la transición de primaria a secundaria de estudiantes indígenas. Más allá de los índices de absorción y eficiencia terminal, es necesario entender mejor los desafíos particulares de los estudiantes. Se requieren estudios que incorporen las voces de maestros, estudiantes y padres de familia, ya que son ellos quienes mejor conocen las condiciones socioeconómicas, lingüísticas, escolares y culturales en las comunidades. Así, los esfuerzos deben dirigirse tanto al mejoramiento de las bases de datos disponibles, como al estudio de los entornos en estrecha colaboración con las comunidades escolares.

El segundo tema tiene que ver con las dificultades que enfrentan los padres para apoyar a sus hijos en secundaria. Si bien los padres de familia que participaron en la investigación señalaron que suelen pedir ayuda a hijos mayores u otros miembros de la comunidad (sobrinos, maestros de primaria), fue evidente que los padres necesitan recursos para apoyar a sus hijos no sólo en labores escolares, sino en motivación y orientación ante los riesgos que enfrentan por estar viviendo cambios físicos y emocionales. Las dificultades sociales, económicas y familiares (migración, familias monoparentales, violencia, padres con poca escolaridad) de los entornos estudiados hacen estos desafíos aún más apremiantes.

Y por último, un tema clave: se requiere un esfuerzo académico y de gestión para la recolección de datos precisos sobre la población indígena en México, pues -como ya se mencionó- no hay indicadores adecuados, representativos ni continuos sobre asistencia y desempeño escolar para la población indígena en general, ni para pueblos indígenas específicos, lo cual genera serias limitaciones para el estudio sistemático del desempeño educativo de los estudiantes indígenas y sus diferencias con respecto a los no-indígenas.

\section{Referencias}

Arenas, E., Rubalcava, L. y Teruel, G. (2016). Social determinants of school continuation in México for the cohort born between 1991- 1993: Evidence using panel data. Documento presentado en la Population Association of America Meeting. Washington, D. C. https://pdfs.semanticscholar.org/cb2d/0803aa4375c428228a175830b42e9ca962d6.pdf

Barrón, C. y Ducoing, P. (2017). La escuela secundaria hoy: problemas y retos. Revista Mexicana de Investigación Educativa, 22(72), 9-30. Recuperado de

https://www.comie.org.mx/revista/v2018/rmie/index.php/nrmie/article/view/2 
Chávez-Herrería, E., Abreu, R. y Charles, A. (2013). Índice de equidad educativa indígena. Informe de resultados para México, sus estados y municipios 2010. México: Fundación Idea, PNUD, SEP. Recuperado de http://fundacionidea.org/posts/34

Fernández, M., Torres, E. y García, C. (2016). Creencias sobre la educación intercultural y prácticas de enseñanza de profesores de secundaria de la población indígena yaqui. Perfiles Educativos, 38(152), 109127.

Gibbs, B. G. y Heaton, T. B. (2014). Dropout from primary to secondary school in Mexico: a life course perspective. International Journal of Educational Development, 36, 63-71.

INEE y UNICEF. (2016). Panorama educativo de la población indígena 2015. México: Autor. Recuperado de http://compromisoporlaeducacion.mx/wp-content/uploads/2016/08/PEPI.pdf

INEE. (2016). Panorama educativo de México. Indicadores del Sistema Educativo Nacional 2015. Educación básica y media superior. México: Autor. Recuperado de

http://publicaciones.inee.edu.mx/buscadorPub/P1/B/114/P1B114.pdf

INEGI. (2013). Censo de Escuelas, Maestros y alumnos de Educación Básica y Especial (CEMABE). Recuperado de http://cemabe.inegi.org.mx

INEGI. (2013b). Encuesta Nacional de Ingresos y Gastos en los Hogares 2013. Recuperado de http://www3.inegi.org.mx/rnm/index.php/catalog/132

Mier y Terán, M. y Rabell, C. (2013). Escolaridad y lengua hablada en comunidades rurales de la península yucateca. Revista Mexicana de Sociología, 75(3), 371-406.

Parker, S., Rubalcava, L. y Teruel, G. (2005). Schooling inequality and language barriers. Economic Development and Cultural Change, 54(1), 71-94.

Tapia, G., Pantoja, J. y Fierro, C. (2010). ¿La escuela hace la diferencia? El abandono de la escuela secundaria en Guanajuato, México. Revista Mexicana de Investigación Educativa, 15(44), 197-225.

Recuperado de https://www.comie.org.mx/revista/v2018/rmie/index.php/nrmie/article/view/428

UNICEF. (2016). Niños y niñas fuera de la escuela. México: Autor.

Van Dijk, S. (2012). La política pública para abatir el abandono escolar y las voces de los niños, sus tutores y sus maestros. Revista Mexicana de Investigación Educativa, 17(52), 115-139. Recuperado de https://www.comie.orq.mx/revista/v2018/rmie/index.php/nrmie/article/view/299

Vargas, E. y Valadez, A. (2016). Calidad de la escuela, estatus económico y deserción escolar de los adolescentes mexicanos. Revista Electrónica de Investigación Educativa, 18(1), 82-97. Recuperado de https://redie.uabc.mx/redie/article/view/713 